\title{
5 Tabu-Thema Rassismus
}

○ Hier geht es zum Glossar.

m Hier geht es zu den Methoden, Artefakten und Werkzeugen.

$\hookrightarrow$ Hier wird das Thema weiter vertieft. 


\section{Kurzfassung}

Während interkultureller ODialog in der Regel nur entsteht, wenn er proaktiv gestaltet und herbeigeführt wird, verhält es sich mit Rassismus anders: Wie viele andere Länder ist Deutschland strukturell von Rassismus geprägt. Er ist - mehr oderweniger verdeckt-allgegenwärtig. Zugleich beschränkt er sich nicht auf absichtlich und bewusst abwertende Äußerungen von Menschen am ,rechten Rand" gegenüber Menschen, die ihrer Meinung nach nicht dazugehören. Rassismus schlägt sich auch regelmäßig in institutionellen Arrangements, alltäglichen Handlungen und Äußerungen nieder, ohne dass er als solcher beabsichtigt oder auf den ersten Blick erkennbar ist. Rassistische Ideologien dienen der Sicherung der Vorherrschaft und OPrivilegien $\bigcirc$,weißer' Menschen. Damit sind sie untrennbar mit Machtfragen verknüpft. Umso wichtiger ist es daher, Rassismus in der Auseinandersetzung mit Barrieren für Interkultur in der Beteiligung nicht zu tabuisieren.

Auch in INTERPART gab es immer wieder Anlässe, über Rassismus nachzudenken. Bei unseren Forschungsaktivitäten kam es zu Begegnungen, die uns irritierten und bei denen wir im Nachhinein das Gefühl hatten, nicht angemessen reagiert zu haben. Gleichzeitig hatten wir Schwierigkeiten, Migrationsgeschichten, Privilegien und Erfahrungen mit Rassismus innerhalb unseres Forschungsteams zu thematisieren und für unsere Forschung zu nutzen.

Wie bei allen Tabu-Themen können wir auch für den Umgang mit Rassismus keine Strategie präsentieren, die immer und überall zum Einsatz kommen könnte und alle zufriedenstellt. Über dieses Thema sprechen viele Betroffene in der Öffentlichkeit ebenso ungern wie Menschen, die sich bisher wenig damit beschäftigt haben. Wo Interkultur ( $\rightarrow$ Kap. 4) eine Zielsetzung gesellschaftlichen Handelns ist, führt jedoch an der expliziten Auseinandersetzung mit rassistischen Erfahrungen kein Weg vorbei - durch das Darüber-Sprechen, Sich-selbst-Hinterfragen und durch Empathie und Reflexivität. Dies kann jede*r für sich 
selbst tun. Olnstitutionen hingegen können Gelegenheiten dafür auch systematisch organisieren, um interkulturellen Dialog, individuelle und kollektive Reflexion anzuregen. 


\section{Erkenntnisse aus der Fachdiskussion}

\section{Rassismus als Tabu-Thema}

Rassismus ist ein Thema, das in der Forschung zu institutionellem Wandel in Verbindung mit Zuwanderung immer mitzudenken ist. Gleichzeitig ist es extrem schwierig anzusprechen-auch oder gerade in der Bundesrepublik (Espahangizi et al., 2016, S. 10). Das liegt daran, dass im Selbstverständnis von Individuen und Institutionen der Begriff Rassismus in der Regel nicht vorkommt (Bukow \& Cudak, 2017). Auch diejenigen, die Vorbehalte äußern gegenüber Zugewanderten oder Menschen, die sie für nicht zugehörig halten, weisen den Vorwurf des Rassismus weit von sich. Sie bestreiten damit gleichzeitig, dass Menschen, die nicht von Rassismus betroffen sind, es ohne eigenes Zutun in vielen gesellschaftlichen Bereichen-z. B. auf dem Wohnungs- oder Arbeitsmarkt - leichter haben und besser behandelt werden als Diskriminierte.

Diese Abwehr hängt u. a. mit der Geschichte des Rassismus in Deutschland zusammen und mit einem engen Rassismusbegriff, der besagt: Rassismus bedeutet, dass,weiße“ Menschen Menschen mit vermeintlich anderer Hautfarbe diskriminieren.

\section{Wandel rassistischer Muster}

Tatsächlich führen viele Menschen heute aber nicht die Hautpigmentierung, sondern kulturelle und/oder religiöse Gründe für Abwertung und Diskriminierung an. Aktuell bedeutet Rassismus häufig, Menschen abzuwerten oder zu benachteiligen, weil ihnen bestimmte Eigenschaften oderVerhaltensweisen unterstellt werden, die auf ihre (vermeintliche oder tatsächliche) Zugehörigkeit 
zu einer ethnischen oder kulturellen Gruppe zurückgeführt werden. Er setzt Menschen in hierarchische Beziehungen zueinander und organisiert Gesellschaft entlang biologischer, ethnischer und kultureller Gruppenzuschreibungen (Tsianos \& Karakayali, 2014, S. 34).

Damit hat ein Wandel stattgefunden von biologistischen (also aufäußerliche Körpermerkmale bezogenen) hin zu kulturalisierenden (also auf Traditionen oder Glauben bezogenen) Diskriminierungsmustern (Kollender, 2016, S. 40). DieserWandel macht es deutlich schwieriger, über Rassismus zu sprechen: Die komplexen und häufig indirekten Formen von institutionellem und Alltagsrassismus sind in diesem Rahmen schwerer zu erkennen (Espahangizi et al., 2016, S.17).

Mit Hautfarbe verbundene Zuschreibungen spielen als rassistisches, kollektiv erlerntes ,Wissen“ in alltäglichen Situationen jedoch nach wie vor eine wichtige Rolle. In Deutschland wird Zugehörigkeit zur Mehrheitsgesellschaft weiterhin häufig über die Gleichsetzung von ,deutsch“ und ,weißer، Hautfarbe definiert; Ähnliches lässt sich in anderen europäischen Ländern beobachten (El-Tayeb, 2015). Auch Namen, die nicht als traditionell deutsch erkannt werden, können zu Diskriminierung führen, z. B. auf dem Wohnungsmarkt (vhw, 2020).

\section{Othering}

Wenn Menschen mit Migrationsgeschichte als Gruppe mit ,anderen'Interessen und Bedürfnissen gegenüber einer Mehrheitsgesellschaft angesprochen werden, wird dies in (post-)kolonialen Debatten als Othering bezeichnet. Der Begriff geht zurück auf das englische,other' (anderes); im Deutschen ist Othering in etwa als ,Fremd-machen' zu verstehen. Othering geschieht häufig aus einer Machtposition heraus: Die eigene Position wird dabei als normal und zugehörig, die andere als besonders und fremd gekennzeichnet. Die Stimmen bestimmter Bevölkerungsgruppen werden damit an die gesellschaftliche Peripherie verdrängt 
(Conrad et al., 2013). Dadurch wird ein Gegensatz zwischen ,normal' und ,anders' konstruiert. Eine vermeintliche deutsche,weiße' Normalität bleibt als Maß der Dinge stabil, das ,Andere“ wird erklärungs- und anpassungsbedürftig.

Zugewanderte und ihre Nachkommen oder Menschen, die nicht ,weiß' sind, als ,abweichend von der Normalität‘ oder ,Andere' zu behandeln, verfestigt soziale Ungleichheiten und verhindert gesellschaftliche OTeilhabe und Pluralität. Dies gilt auch dann, wenn das Othering aus dem expliziten Wunsch resultiert, zugunsten bestimmter Zielgruppen zu handeln. Damit sind, absichtlich oder nicht, Othering und Rassismus potenziell miteinander verbunden (Ahmed, 2012). Aktuelle Forschungen zu Rassismus zeigen, dass rassistische Denkmuster in vielen Köpfen und Strukturen tief verankert sind. Sie werden - teils unabsichtlich-kontinuierlich reproduziert, sogar von Menschen, die Rassismus aktiv bekämpfen wollen (Weiß, 2013).

\section{Rassismus und Beteiligung}

Auch in der Beteiligungspraxis für die Stadtentwicklung laufen zielgruppenspezifische Angebote für bestimmte Bevölkerungsgruppen Gefahr, deren ,Anderssein' fortzuschreiben. Sie können aber auch positive Effekte für die Repräsentation und Teilhabe bestimmter Gruppen erreichen (Young, 2005), da sie ein Forum und ggf. geschützte Räume für diese Gruppen schaffen. Zudem unterstützen sie sie dabei, ihren Anliegen Gehör zu verschaffen.

Das Gefühl, einer gesellschaftlich definierten Normalität nicht zu entsprechen, kann ein Grund für geringes Interesse oder Unsicherheit im Rahmen von Beteiligungsverfahren sein (vhw, 2018). Rassistische Diskriminierung und die Erfahrung, diese nicht thematisieren zu können, weil Nicht-Betroffene sie nicht ernst nehmen oder abstreiten, verhindert einen offenen Austausch auch in Beteiligungsprozessen.

Wie bereits in $\hookrightarrow$ Kapitel 4 diskutiert, unterscheiden sich migrantische 0 Milieus in zeitgenössischen postmigrantischen 
Stadtgesellschaften hinsichtlich ihrer Vielfalt an Lebensstilen, Bildungsorientierung, Konsumverhalten, religiöser Praxis und auch ihres Interesses oder Desinteresses an Beteiligung kaum von jenen der Menschen ohne Migrationsgeschichte (vhw, 2018). Insofern besteht die Gefahr, durch Othering nicht nur künstliche Gegensätze zwischen Menschen zu schaffen oder aufrechtzuerhalten, sondern auch rassistisch geprägte Vorstellungen in der Stadtgesellschaft und ihren Institutionen fortzuschreiben.

\section{Begegnungen mit Rassismus in den INTERPART- Reallaboren}

Im Kontext von Interkultur sahen wir es als erforderlich an, auch eine Reflexion über Rassismus in der Beteiligung anzuregen und Zusammenhänge zwischen Rassismus in den Institutionen und Beteiligungsbarrieren zu betrachten.

\section{Alltägliches Othering}

Gleich zu Beginn von INTERPART stießen wir in zwei Begegnungen ganz beiläufig auf das Thema Othering.

In einem der ersten DFachworkshops in den Verwaltungen berichtete eine Teilnehmerin von einem Gespräch, das sie am Rande einer Beteiligungsveranstaltung mit einer Stadtnutzerin geführt hatte. Die Frau habe ,südländisch“ ausgesehen und perfekt Deutsch gesprochen. Im Laufe des Gesprächs habe sie sich beschwert, dass sie ständig gefragt werde, woher sie komme und wann sie zurückgehe, obwohl sie in Deutschland aufgewachsen sei. Die Verwaltungsmitarbeiterin beendete ihren Bericht mit den 
Worten: „Und mit solchen Befindlichkeiten muss man sich dann auch noch beschäftigen" (\#FWS1_WI).

Bei der Vorbereitung eines DPPodcast-Dialogs hakte eine ,weiße' deutsche Teilnehmerin (It. eigener Aussage ohne Migrationsgeschichte) ebenfalls nach, ob man denn nicht mehr fragen dürfe, woher jemand komme. Nicht alle Menschen mit Migrationsgeschichte haben Lust, darüber zu sprechen - vor allem dann, wenn auf die Antwort, man sei aus X-Stadt, schon öfter nochmals nachgefragt wurde, woher man denn nun ,wirklich“ komme, wie auch der folgende Bericht einer Podcast-Teilnehmerin zeigt:

„Das ist das Problem: Wer fragt, woher man kommt?
Ich sage gerne, ich komme aus Mainz, was auch
stimmt. Dann wird gesagt: ,Nein, ich meine das an-
ders.' Dann frage ich öfters: ,O.k., dann erzähl mir
doch mal: Was genau möchtest du denn wissen?‘ Es
ist mehr als woher man kommt. Möchte ich immer er-
zählen, woher ich komme? Das ist irgendwie immer
mit der Vergangenheit verbunden. Man wird zu einer
Kultur gemacht, in der man fünf Jahre aufgewachsen
ist-oder gar nicht in vielen Fällen. Aber dann redet
man immer über die Vergangenheit und nicht über
das Jetzt und dass man von mir erwartet, dass ich
eine [bestimmte] Kultur in mir vertrete, was über-
haupt nicht der Fall ist. Die ist auch so unterschied-
lich.“ (\#SD_3_Pg)

In einem MExpertinnen-Interview mit einer Verwaltungsmitarbeiterin mit Migrationsgeschichte wurde eine ähnliche Situation aus der Perspektive des beruflichen Alltags in der Verwaltung geschildert:

\footnotetext{
„Aber diese Fragen muss ich mir auch gefallen lassen, selbst als Mitarbeiterin der Verwaltung. Wenn ich dann sage, ich fahr jetzt in die Türkei, heißt es: ,Ach, Du fährst in die Heimat" - wo ich denke, nee, die hat gar nichts verstanden [...]. Früher hätte ich mich da
} 
[...] aufgeregt, ja. Das sagt man dreimal, und wenn die dann immer noch davon sprechen: ,Ach, Du fährst in die Heimat;, dann denke ich mir, okay, hab keinen Bock mehr [...]. Da ist man einfach müde auch irgendwann [...]. Für die einen ist es ein Luxus, sich damit auseinanderzusetzen, und für die anderen ist es irgendwie tagtägliche Erfahrung." (\#sh_001)

Viele Betroffene empfinden diese Fragen als Othering, die den Zweck verfolgen, dass sich die fragende Person - absichtlich oder unabsichtlich - der ,Fremdheit' des Gegenübers und der eigenen ,Normalität' vergewissert. Es macht einen großen Unterschied, wenn hingegen ehrliches Interesse an der anderen Person erkennbar wird.

\section{Denkraum}

Woher kommen Sie? Wieviel oder wie wenig hat Ihre

Herkunft damit zu tun, wer Sie heute sind? Was

werden andere über Sie denken, wenn sie hören,

woher Sie kommen? Stimmt das mit Ihrem

Selbstbild überein?

\section{Das Privileg der Normalität}

Für die einen sind die o. g. Geschichten,Befindlichkeiten', und sie sind erstaunt und irritiert von der Idee, darauf mit Reflexivität, Empathie und Rücksicht reagieren zu sollen. Sehr überspitzt formuliert: Muss man auf jede individuelle,Befindlichkeit' Rücksicht nehmen? Für andere sind solche ,Befindlichkeiten` alltägliche, gelegentlich sogar traumatische Erfahrungen der Ausgrenzung 
und Verletzung. Interkultureller Dialog erfordert deshalb die Reflexion von Machtverhältnissen, die sich in der Beteiligung als Rassismus - sowohl in Institutionen als auch in persönlichen Haltungen - und als ,weiße Privilegien‘ zeigen (Dyer, 2005).

Auch in INTERPART begegneten uns diese Fragen. Ein Beispiel: Wir hatten unterschiedliche lokale Initiativen eingeladen, mit uns zu diskutieren, wie gemeinsam über Stadtentwicklung gesprochen werden könnte. Diese sehr akademische Frage sprach - wie uns leider erst vor Ort klar wurde - vor allem Teilnehmer*innen mit einem akademischen, nicht-migrantischen Hintergrund an. Im Laufe des Workshops wurde deutlich, dass die Teilnehmer*innen den Workshop als einen Ort definierten, an dem ausgrenzende Äußerungen von allen geteilt und nicht sanktioniert würden. Die Moderation war darauf nicht vorbereitet und in dem Moment auch nicht in der Lage, angemessen zu reagieren. Das Forschungsteam war irritiert, wollte aber die anwesenden Ko-Forscher*innen und Stadtnutzer*innen nicht vor den Kopf stoBen, um die weitere Zusammenarbeit mit ihnen nicht zu so einem frühen Zeitpunkt schon zu belasten. Gleichzeitig hatten wir das Gefühl, durch unsere Zurückhaltung eigene Integrität in diesem Bereich einzubüßen.

Im Nachhinein diskutierten wir mögliche Wege, um Rassismus im weiteren Projektverlauf entgegenzutreten. Wir waren uns einig, dass wir diskriminierte Stimmen unterstützen und einen Rahmen schaffen wollten, um Rassismus explizit zu machen, wenn er uns begegnet. Dabei wurde uns erneut bewusst, dass Otransformative Forschung ebenso politisch ist wie Beteiligung und dass wir naiv gewesen waren anzunehmen, uns in einem unbekannten Umfeld schnell hinsichtlich unserer Reallabor-Aktivitäten orientieren zu können. Gebraucht werden Formate und Personen, die Positionen von rassistisch Diskriminierten unterstützen. Diesen normativen Ansatz versuchten wir im Podcast zu realisieren.

Auch bei der partizipativen Podcast-Produktion kam das Thema Rassismus auf. Zentral war auch hier die Frage zur Deutungshoheit im Stadtteil: Wer hat das Recht, über die Zugehörigkeit zu einem Stadtteil etwas zu sagen? Hier achteten die 
Forscher*innen bei der später eingesprochenen Moderation und dem Schnitt der Tonspur besonders darauf, dass marginalisierte Positionen mindestens gleichberechtigt erzählt wurden. Dem Ergebnis stimmten alle Beteiligten zu.

\section{Denkraum}

Mit welchen anderen Formaten könnten ähnlich wie bei unserer Podcast-Produktion von Rassismus betroffene Personen und ihre Perspektiven im Kontext von Beteiligung aktiv gestärkt werden? Glauben Sie, diese Formate haben Potenzial für die Beteiligung in der Stadtentwicklung?

\section{Interkulturelle Verständigung über Rassismus}

In Expert*innen-Interviews mit Verwaltungsmitarbeiter*innen und Fachworkshops kamen im Zusammenhang mit Rassismus in den Institutionen weitere Faktoren zur Sprache. Dazu zählten die fehlende Diversität des Verwaltungspersonals, eine Unterrepräsentation von Migrant*innen in Leitungspositionen sowie die Vorherrschaft der deutschen (Amts-)Sprache in der Kommunikation.

Um Rassismus in Institutionen explizit zu besprechen, fanden ein Online-מ.Fachdialog „Rassismus in den Institutionen“ mit Vertreter*innen aus Verwaltung und OZivilgesellschaft sowie eine Online-Gruppendiskussion mit zivilgesellschaftlichen Initiativen statt. Im Online-Fachdialog Rassismus betonte ein Teilnehmer aus der Verwaltung deren besondere Verantwortung: 


\begin{abstract}
„Wir haben da natürlich auch einen besonderen Blick, denke ich, auf Rassismus. Institutionen sind zweifelsfrei meines Erachtens rassistisch, und das betrifft alle Handlungen einer Institution. Alles andere zu behaupten wäre, glaube ich, auch nicht realistisch, weil die Gesellschaft sich strukturiert nach Race, Class, Gender und dies über die gesellschaftlichen Machtverhältnisse entscheidet. Also Rassismus macht natürlich vor der Tür [der planenden Verwaltung] auch nicht halt.“ (\#FD_RI)
\end{abstract}

Auch stand die Frage im Raum: Wie können wir über Barrieren in der Beteiligung sprechen, die durch strukturellen Rassismus in staatlichen Institutionen entstehen? Um diese Auseinandersetzung gemeinsam zu führen, waren die vielseitigen Perspektiven in den Verwaltungen, aber auch in der Zivilgesellschaft wichtig. Dazu gehört das Nachdenken über Privilegien ,weißer' Menschen, die im Alltag nicht von rassistischen Diskriminierungen betroffen sind, wie es z. B. diese Verwaltungsmitarbeiterin in einem Fachdialog formulierte:

„Die Schuld wird immer bei den einzelnen Menschen, dort bei den migrantischen Menschen gesucht und nicht bei der Institution, die die Aufgabe hat, gesellschaftliche Teilnahme sicherzustellen. Das hat etwas mit Haltung zu tun, und ich glaube, das ist die [...] Gefahr, dass wir die Probleme bei anderen sehen und weniger davon ausgehen, dass die Institution dafür zuständig ist, Teilhabe zu gewährleisten.“ (\#FD_RI)

Beispiele dafür sind Zuschreibungen von Partizipationsdefiziten bei Geflüchteten. Das geschieht z. B. in der Annahme, diese seien schwer ansprechbar für Beteiligung, weil sie in ihren Ländern nie Demokratie gelernt hätten, oder sie seien aufgrund ihrer Familienorientierung schwerer für zivilgesellschaftliches Engagement mobilisierbar. 
Struktureller Rassismus ist aus Sicht aller Beteiligten eine zentrale Barriere, die Teilhabe- und Beteiligungsmöglichkeiten einschränkt. Die Vertreterin eines Ointermediären Trägers berichtete von ihren Erfahrungen, dass Geflüchtete bei Behörden eher Hilfe bekommen, wenn sie in Begleitung einer ,weißen“ Person kommen. Auch der INTERPART-Ansatz wurde durchaus kritisch gesehen: Es sei besser, mit dem Begriff Milieu zu arbeiten, denn ,Kultur' sei ein schwer zu fassender Begriff.

Teilnehmer*innen aus den Verwaltungen betrachteten migrantische Selbstorganisationen als wichtige Partner*innen, um fortlaufend zum Thema Rassismus sensibilisiert zu werden. Sie wiesen darauf hin, dass es in vielen Fachämtern noch an einer solchen Sensibilisierung mangele. Interne Weiterbildungsangebote würden sich überwiegend auf einen positiven Umgang mit gesellschaftlicher Vielfalt beziehen, Rassismus dabei hingegen eher ausblenden. Die Personalentwicklung wurde als wichtiges Instrument genannt, um gezielt Menschen in die Verwaltung zu holen, die selbst Erfahrungen mit Rassismus gemacht haben.

Eine Verwaltungsmitarbeiterin plädierte darüber hinaus für einen personellen Umbau der Verwaltung, z. B. durch Quotierungen, um die Gesellschaft besser abzubilden. Vertreter*innen zivilgesellschaftlicher Organisationen machten ebenfalls strukturellen Rassismus dafür verantwortlich, dass Menschen mit Migrationsgeschichte vielerorts in den Verwaltungen unterrepräsentiert sind. Die Gründe dafür ließen sich jedoch nicht nur auf eine ,weiße‘ institutionelle Kultur reduzieren. Das,Wissen über Rassismus' der von Rassismus Betroffenen werde gesamtgesellschaftlich nicht genug thematisiert. Aus Sicht einer Teilnehmerin an den Erzählformaten ( $\hookrightarrow$ Kap. 6 ) stellte sich die Situation z. B. so dar:

„Miteinander-ja, das ist noch schwierig, wenn ich ganz ehrlich bin [...] Wenn man irgendwo hingeht [...], da sind immer noch die Gruppen, sage ich jetzt ganz ehrlich [...]. Ich meine, ich habe auch einen deutschen Pass. Ich war drei Jahre alt, als ich hierherkam mit meiner Mama. Ich bin jetzt 44, also 41 Jahre bin ich schon hier. Ich habe hier alles gemacht: meine Schul- 
bildung, Ausbildung, alles. Aber trotzdem meckert man, so: „Wieso kannst Du Dich nicht integrieren? Aber da müssen beide Seiten mitmachen. Wenn [...] die [mit Migrationsgeschichte] sich jetzt nur, sage ich jetzt mal, die Mühe machen und die anderen sagen: ,Nein, wollen wir nicht' - Da müssen schon beide Seiten mitmachen.“(\#hs_016)

\section{Rassismus oder Nostalgie? Begegnungen in den Vor-Ort-Interventionen}

Bei einer der ersten $\mathbf{m V o r - O r t - I n t e r v e n t i o n e n ~ k a m ~ e i n ~ A n w o h n e r ~}$ vorbei und wollte die Gelegenheit nutzen, um sich zu beschweren. Für ihn hatte sich der Stadtteil in den vergangenen Jahren nachteilig entwickelt. Diese negative Entwicklung macht er exemplarisch fest am Verlust des,deutschen Metzgers', an dessen Stelle ein türkischer Supermarkt eröffnet hatte. In der Auswertung der ФErzählecke bei derselben Vor-Ort-Intervention kamen diese und ähnliche Geschichten über den Wandel der GeschäftsstraBen im Stadtteil immer wieder auf: Früher habe es traditionelle deutsche Geschäfte gegeben, heute gebe es überwiegend türkische und griechische Geschäfte (\#rb_012).

Solche persönlichen Erzählungen sind natürlich sehr subjektiv. Bei der wissenschaftlichen Auswertung mussten wir deshalb abseits des reinen gesprochenen Worts auch die Positionierung derWissenschaftlerin und der Erzähler*innen und vor allem das Verhältnis zwischen ihnen berücksichtigen. Erzähler*innen richten ihre Geschichten an ihre Zuhörer*innen, und so unterscheiden sich Geschichten je nach Gesprächssituation und Publikum. Umgekehrt spielt Positionierung auch bei der Auswertung eine Rolle: Alle Forscher*innen lesen und interpretieren eine Erzählung etwas anders.

Aus diesem Grund gaben wir unsere Interpretationen an die Teilnehmer*innen zurück und stellten unsere Analysen zur Disposition. So fand in Biebrich ein Validierungsworkshop statt, in 
KAPITEL 5: TABU-THEMA RASSISMUS

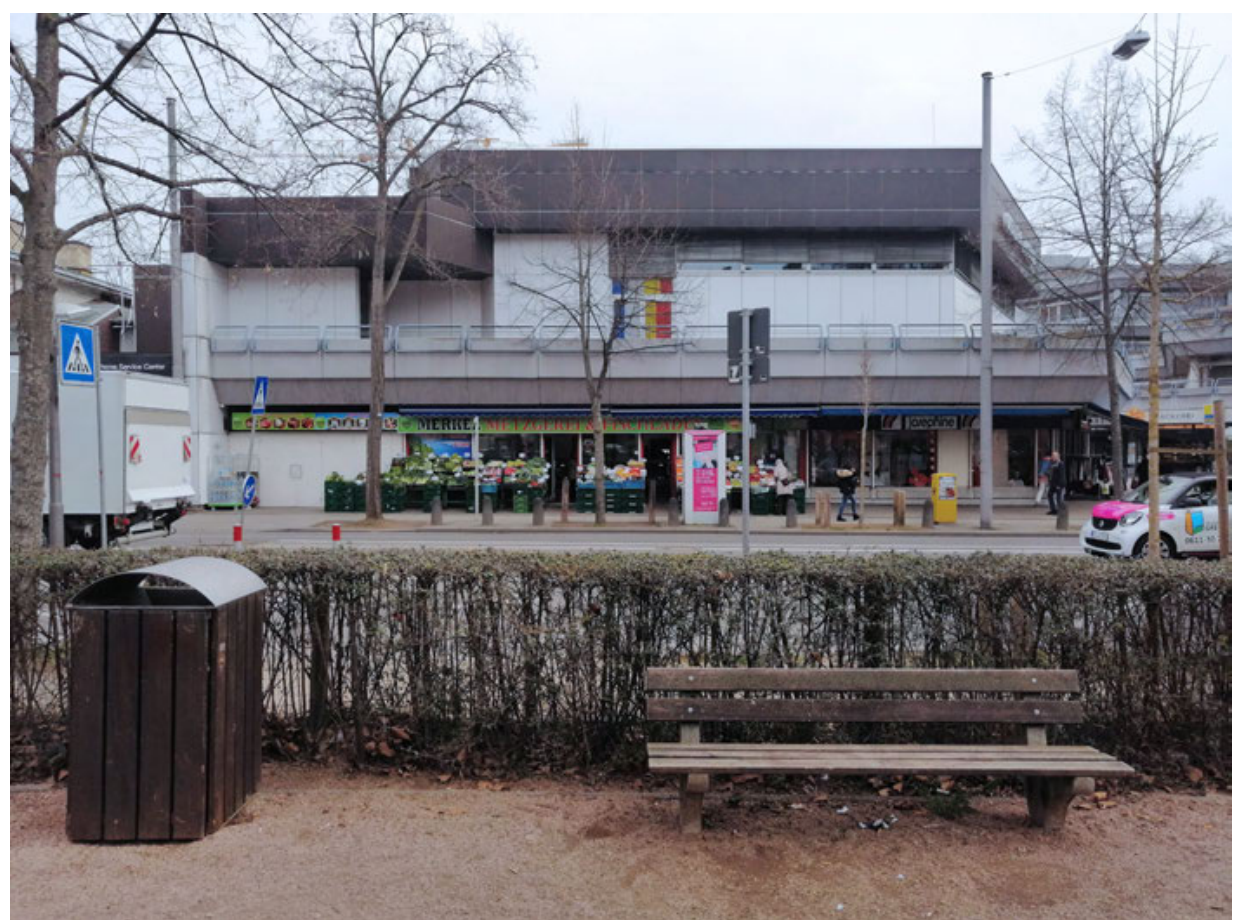

Metzgerei an der Galatea-Anlage, Wiesbaden-Biebrich 
dem das Vorgehen bei der Analyse und unsere Interpretationen dieser und anderer Erzählungen diskutiert wurden. Unser Befund war, dass die Trennlinien der Perspektiven entlang des Vorhandenseins bzw. Nichtvorhandenseins einer Migrationsgeschichte verlaufen. Darin erkannten wir (auch) rassistische Denkmuster.

Im Validierungsworkshop fragten wir die Stadtnutzer*innen nach ihren Wahrnehmungen. Die Teilnehmer*innen griffen unseren Befund auf, stellten aber die Storyline gleichzeitig in einen breiteren Kontext: Die Diskussion um die zunehmende Zahl an Friseuren und Barber-Shops sei eine Diskussion von Deutschen ohne Migrationsgeschichte. Aber Rassismus sei eine zu negative Beschreibung dessen, was im Stadtteil passiere. Ältere Menschen hätten immer auch eine Erinnerungsaufgabe, um Traditionen zu bewahren und zwischen Generationen zu kommunizieren. Alte Traditionen und somit traditionelle Geschäfte bewahren zu wollen, habe erst einmal seine Berechtigung. Sobald allerdings der (nationale) Hintergrund des Ladenbesitzers störe, dann sollte man dies als Rassismus benennen. Veränderungen hätten immer mit Angst zu tun, Zugehörigkeit zu verlieren. Das Narrativ ,Früher war alles besser' ist in vielen Kontexten verbreitet und hat auch mit einem (drohenden) Verlust an Deutungshoheit zu tun.

Diese Hinweise halfen uns, die Erzählungen in einen breiteren Kontext zu setzen und Konflikte besser zu verstehen. Die Interpretation von Erzählungen aus einem Stadtteil denjenigen Menschen zurückzugeben, die dort leben, erwies sich als wichtig. Es verhinderte, falsche Schlüsse zu ziehen, und ließ uns Konflikte unter der Oberfläche besser erkennen. 
KAPITEL 5: TABU-THEMA RASSISMUS

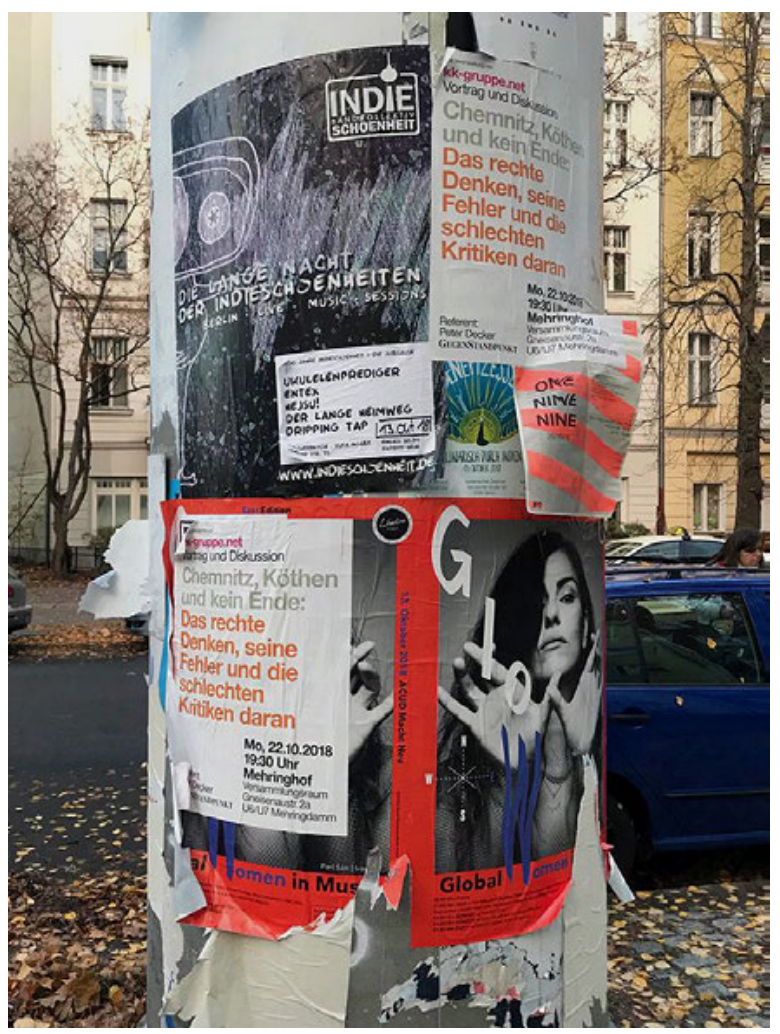

Litfaßsäule, Berlin-Moabit 


\section{Reflexion}

Das Reden über Rassismus und Privilegien ist eine komplexe Aufgabe. Sie zu bearbeiten kann ein Startpunkt sein, um Institutionen interkulturell zu öffnen ( $\hookrightarrow$ Kap. 8) und Barrieren in der Beteiligung ( $\rightarrow$ Kap. 3) zu identifizieren. Allerdings ist es nicht leicht, über das Thema zu sprechen. Die rassistisch motivierten Anschläge in Halle/ Saale und Hanau haben auch in Deutschlandein größeresBewusstsein für das Thema erzeugt. Auch die Black-Lives-Matter-Bewegung hat medial eine größere Aufmerksamkeit für unterschiedliche Perspektiven auf Rassismus generiert, als dies noch vor einigen Jahren der Fall war. Insofern öffnet sich gerade ein Gelegenheitsfenster, entsprechende Reflexionsräume bewusst zu schaffensowohl im städtischen Raum als auch in den Institutionen.

Es stellt sich die Frage: Was können und wollen Planungsverwaltungen leisten, um von einer dominanten und kaum reflektierten ,weißen' Perspektive wegzukommen? Was, um die benachteiligenden Effekte von Privilegien und Wissenshierarchien anzuerkennen? Interkulturelle Beteiligung ( $\hookrightarrow$ Kap. 3 und Kap. 4) stößt auf unüberwindbare Barrieren, wenn Rassismus ein Tabu-Thema bleibt. Um Interkultur tatsächlich umzusetzen, ist ein Austausch zu diesen Fragen zwischen Verwaltungen, Initiativen oder einzelnen Betroffenen nötig.

Beteiligungsverfahren sollten darüber hinaus Mechanismen enthalten, die rassismuskritische und marginalisierte Positionen unterstützen und dabei helfen, Abwertung oder Banalisierung von Diskriminierungserfahrungen zu vermeiden. Der Versuch, über einen differenzierten Milieu-Ansatz mehr Menschen für interkulturelle Beteiligung ( $\hookrightarrow$ Kap. 3 und Kap. 4) zu erreichen, deutet bereits an, dass es in Zukunft nicht nur darum gehen kann, eine größere Zahl von Menschen zu aktivieren. Ein weiteres Ziel wäre es, marginalisierten Positionen, z. B. von rassistisch stigmatisierten Gruppen, aktiv Gehör zu verschaffen. Dadurch würde gleichberechtigte Vielfalt in der Beteiligung zumindest potenziell ein Stück weit normalisiert - gebunden an gleiche Möglichkeiten, Rechte und Pflichten. 